\title{
Crystal, Magnetic and Dielectric Studies of the 2D Antiferromagnet: $\mathrm{\beta}-\mathrm{NaMnO}_{2}$
}

\author{
Ioanna Bakaimi, ${ }^{1,2}$ Artem Abakumov, ${ }^{3}$ Mark A. Green, ${ }^{4,5}$ Alexandros Lappas ${ }^{1}$ \\ ${ }^{1}$ Institute of Electronic Structure and Laser, Foundation for Research and Technology - Hellas, \\ Vassilika Vouton, 71110 Heraklion, Greece \\ ${ }^{2}$ Department of Physics, University of Crete, Voutes, 71003 Heraklion, Greece \\ ${ }^{3}$ EMAT, University of Antwerp, Groenenborgerlaan 171, B-2020 Antwerp, Belgium \\ ${ }^{4}$ NIST Center for Neutron Research, 100 Bureau Drive, Gaithersburg, MD 20899-8562, USA \\ ${ }^{5}$ School of Physical Sciences, University of Kent, Canterbury, Kent CT2 7NH, UK
}

\begin{abstract}
In this paper we present our recent studies on the crystal, magnetic and dielectric properties of the $\beta-\mathrm{NaMnO}_{2}$. Experimental results of neutron powder and electron diffraction combined with measurements of the dielectric permittivity suggest that the $\beta-\mathrm{NaMnO}_{2}$ is an excellent candidate for studying the coupling between the magnetic and electric degrees of freedom. Neutron powder diffraction data reveal the existence of a commensurate and an incommensurate magnetic structure at $200 \mathrm{~K}$ and below $100 \mathrm{~K}$, respectively. Dielectric anomalies which appear at the temperature regions where the two magnetic structures emerge, indicate the appearance of magnetodielectric coupling.
\end{abstract}

Keywor ds: magnetic frustration, neutron powder diffraction, dielectrics, magneto-electric coupling.

\section{INTRODUCTION}

The coupling between the magnetic and electric degrees of freedom has been proved to be a particularly appealing subject of research, since the breathtaking revival of the magneto-electric effect. This phenomenon was firstly realized on the $\mathrm{Cr}_{2} \mathrm{O}_{3}$ in the early 1960's ${ }^{[1]-[4]}$ and has triggered a tremendous interest for two reasons. The first one arises from the need to address the theoretical frame and to describe the magnetic symmetry considerations which would explain the mechanisms that generate and allow the phenomenon. The second reason is the potential for technological applications such as multifunctional devices. The magnetoelectric effect allows the control of the magnetic properties via an external electric field and vice versa.

Most of the compounds that exhibit the magneto-electric coupling belong to the perovskite family, such as the $\mathrm{TbMnO}_{3}{ }^{[5]}$ the $\mathrm{YMnO}_{3}{ }^{[6]}$ In "type II multiferroics", widely known as magnetic ferroelectrics, ${ }^{[7],}$, [8] the ferroelectric order appears below the temperature of the magnetic transition. Nevertheless, the explanation of the underlying mechanism is not only based on the appearance of specific spin configurations such as cycloid or proper, but requires also a complete understanding of the crystal and magnetic symmetry. The recent discovery that the magnetic frustration is related to the appearance of the magnetoelectricity, ${ }^{[9]}$ lead to the study of the compounds with the chemical formula $\mathrm{ABO}_{2}$ ( $\mathrm{B}$ is a $3 \mathrm{~d}$ magnetic ion such as $\mathrm{Fe}, \mathrm{Mn}$, and $\mathrm{A}$ : non magnetic ion usually $\mathrm{Na}, \mathrm{Li}, \mathrm{Cu}$ ) as possible magnetoelectrics. Indeed, the compounds $\mathrm{CuFeO}_{2},{ }^{[10]} \mathrm{ACrO}_{2}(\mathrm{~A}: \mathrm{Cu}, \mathrm{Cr})^{[11]}$ and $\mathrm{AgFeO}_{2}{ }^{[12]}$ have been reported to exhibit the magnetoelectric coupling. Most interestingly, it has been reported that the mechanism, which explains the phenomenon in these compounds, is not related to the Dzyaloshinskii Moriya interaction, ${ }^{[13]-[15]}$ as it happens in several magnetoelectrics. Recent studies suggest that the underlying mechanism of the magnetoelectric coupling in the $\mathrm{ABO}_{2}$ compounds is the spin orbit interaction of the d-p hybridization. [16], [17]

In view of the possible magneto-electric coupling of the $\beta-\mathrm{NaMnO}_{2}$ we have studied the crystal, magnetic and dielectric properties of the compound. $\beta-\mathrm{NaMnO}_{2}\left(\mathrm{Mn}^{+3}, 3 \mathrm{~d}^{4}, \mathrm{~S}=2\right)$, belongs to a vast family of oxides, namely the $\mathrm{Na}_{\mathrm{x}} \mathrm{MnO}_{2}$ oxides, which has been first reported by Parant et al . ${ }^{[18]}$ The $\mathrm{Na}_{\mathrm{x}} \mathrm{MnO}_{2}$ have been widely studied as initial precursors in chemical reactions which provide various modified layered structures such as the birnessite structure. ${ }^{[19]-}$ ${ }^{[21]}$ Moreover $\mathrm{Na}_{x} \mathrm{MnO}_{2}$ have attracted a lot of industrial interest due to their possible applications as cathode materials 
in rechargeable batteries. ${ }^{[22]-[24]}$ In the $\beta-\mathrm{NaMnO}_{2}$, the $\mathrm{Mn}$ cations map out on a corrugated 2D triangular lattice, whereas the $\mathrm{MnO}_{6}$ edge-sharing octahedra are separated by two layers of $\mathrm{Na}$ cations (figure 1). The triangular arrangement of the $\mathrm{Mn}$, is favorable for the existence of competing magnetic interactions between the magnetic cations, as it has been proved for the case of the $\alpha-\mathrm{NaMnO}_{2}$. ${ }^{[25]-[28]}$

A

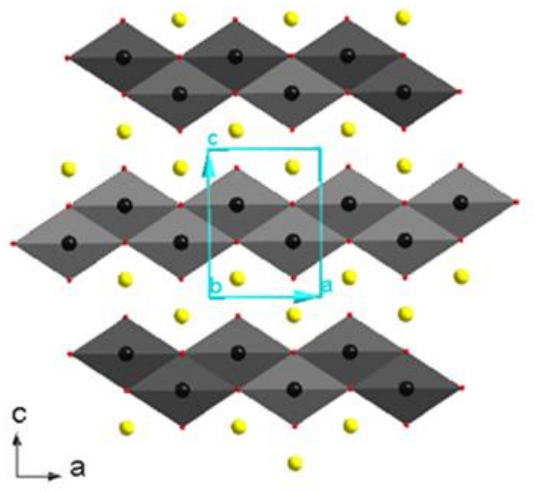

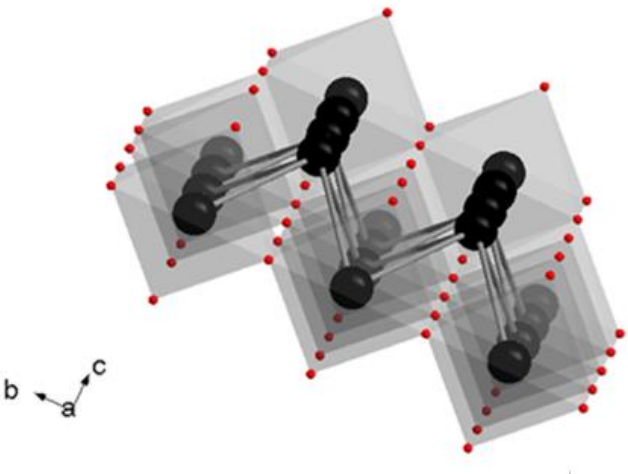

Figure 1.The crystal structure of the $\beta-\mathrm{NaMnO}_{2}$. The black, red and yellow spheres represent the manganese, oxy gen and sodium atoms, respectively. Figure 1a shows the lay ered structure, composed by sheets of $\mathrm{MnO}_{6}$ edge sharing octahedra separated by two layers of sodium cations. The aqua lines represent the cell edges. The 2D triangular arrangement of the Mncations is shown in figure $1 \mathrm{~b}$.

\section{EXPERIMENTAL PART}

\subsection{Sythesis of polycrystalline powder of $\beta-\mathrm{NaMnO}_{2}$}

Polycrystalline powder specimens of $\beta-\mathrm{NaMnO}_{2}$ have been synthesized by using high temperature solid state reaction at $950^{\circ} \mathrm{C}$. The synthesis is based on the $\beta-\mathrm{NaMnO}_{2}$ synthetic protocol reported by Parant. [18]

\subsection{Characterization of $\beta-\mathrm{NaMnO}_{2}$}

The purity and crystallinity of the $\beta-\mathrm{NaMnO}_{2}$ phase has been checked by X-Ray diffraction on a Rigaku D/MAX$2000 \mathrm{H}$ rotating $\mathrm{Cu}$ anode diffractometer $(\lambda=1.5406 \AA)$. The sample is placed on the holder and sealed with a mylar foil in the glove box, so that it is not exposed in the atmosphere during the $\mathrm{X}$-Rays experiment. The synthesized compound is a phase-pure $\beta-\mathrm{NaMnO}_{2}$ which crystallizes in the orthorhombic Pmnm symmetry $(a=2.852 \AA, b=6.33 \AA$, $c=4.77 \AA$, $90^{\circ}, 90^{\circ}, 90^{\circ}$ ). Thermogravimetric measurements were carried out on a SDT-Q600 while the sample was heated with a constant heating rate of $10^{\circ} \mathrm{C} / \mathrm{min}$ under Argon atmosphere. Neutron powder diffraction experiments took place on the BT1 diffractometer, with the wavelength of $2.0787 \AA$ at the NCNR-NIST, USA. High resolution powder diffraction measurements were conducted on the $11 \mathrm{BM}$ beamline, of the Argonne National Laboratory, in USA using the wavelength of $0.41395 \AA$. The dielectric constant and dielectric loss, have been measured on $5 \mathrm{~mm}$ pellets of $\beta$ $\mathrm{NaMnO}_{2}$ by an Agilent 4284 LCR bridge (at $1 \mathrm{~V}$ or $2 \mathrm{~V}$ oscillation voltage amplitude) at the temperature range between $77 \mathrm{~K}-300 \mathrm{~K}$. An average pressure of $6.5 \times 10^{8} \mathrm{~Pa}$ was applied with a $5 \mathrm{~mm}$ homemade steel press in the glove box in order to form the $\beta-\mathrm{NaMnO}_{2}$ pellets. Silver paste contacts have been made on the $5 \mathrm{~mm} \beta-\mathrm{NaMnO}_{2}$ pellets which would be used in the magneto-dielectric measurements. The samples were placed in Janis Cryostat of continuous nitrogen flow and cooled starting from room temperature to $77 \mathrm{~K}$ under zero electric and magnetic field. The capacitance and dielectric loss values were recorded upon heating at various frequencies. The calculation of the real part of the dielectric permittivity $\varepsilon^{\prime}$ has been done using the equation $\varepsilon^{\prime}=\varepsilon_{0} \mathrm{Cg}$, where $\varepsilon_{0}$ is the dielectric permittivity of the vacuum $\left(8.85 \times 10^{-12} \mathrm{~F} / \mathrm{m}, \mathrm{C}\right.$ is the capacitance $(\mathrm{F})$ and $\mathrm{g}$ is the geometric factor $(\mathrm{m})$ of the sample calculated by dividing the conductive area of the sample (A) with its thickness (d). 


\section{RESULTS AND DISCUSSION}

The $\beta-\mathrm{NaMnO}_{2}$, crystallizes in the orthorhombic structure at room temperature. It has been indexed upon the orthorhombic structure ${ }^{[18]}$ (Space-group Pmnm a=2.852(3) $\AA, b=6.31(2) \AA \mathrm{c}=4.77(1) \AA$, ICSD 16271) as shown in figure 2. Red ticks correspond to the indexing based on the orthorhombic cell, whereas the broad intense peak between $20 \mathrm{deg}-30 \mathrm{deg}$ and the one at $38 \mathrm{deg}$ pointed with the grey asterisk are attributed to the mylar film used in order to protect the sample from moisture. The peak at $16.68 \mathrm{deg}$ pointed with the blue tick corresponds to the 001 peak of the a$\mathrm{NaMnO}_{2}$ phase. An unusual characteristic observed at the X rays Powder Diffraction (XRPD) pattern is the broadening of the peaks at 38-40deg and more clearly at $55 \mathrm{deg}$.

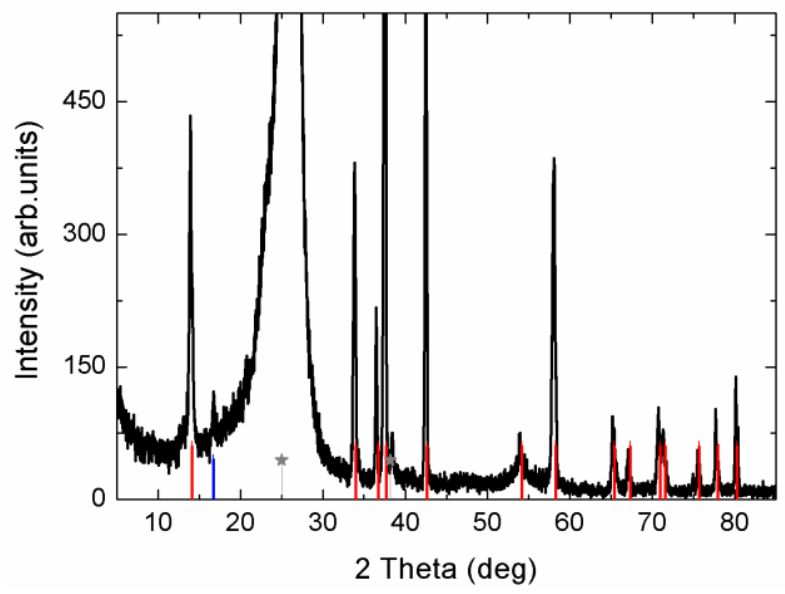

Figure 2. Room temperature $\mathrm{XRD}$ of the $\beta-\mathrm{NaMnO}_{2}$ polycrystalline powder indexed upon the orthorhombic cell. Red ticks correspond to the indexing of the orthorhombic cell of $\beta-\mathrm{NaMnO}_{2}$. The blue tick at 16.68 deg corresponds to the 001 peak of the monoclinic cell of $\alpha-\mathrm{NaMnO}_{2}{ }^{[26]}$ The peaks shown with the grey asterisks at $25 \mathrm{deg}$ and 38 deg are attributed to the my lar film.

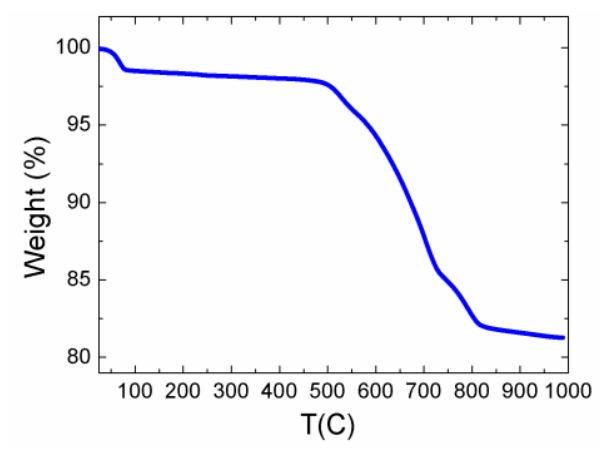

Figure 3. A graph showing weight loss (\%) versus temperature for $\beta-\mathrm{NaMnO}_{2}$.

In order to evaluate the stability of the $\beta-\mathrm{NaMnO}_{2}$ phase in respect with the temperature, thermogravimetric measurements were carried out in the temperature range between $25^{\circ} \mathrm{C}$ and $1000^{\circ} \mathrm{C}$ (figure 3 ). The initial weight decrease of less than $1.5 \%$ around $100^{\circ} \mathrm{C}$ is attributed to the evaporation of water, which could be absorbed by the pores of the sample's surface while transferring the sample out of the glove box to the alumina pan of the SDT-Q600. The chemical composition of the sample remains stable at the temperatures $100^{\circ} \mathrm{C}-500^{\circ} \mathrm{C}$, whereas the $16 \%$ weight loss noticed between $500^{\circ} \mathrm{C}-800^{\circ} \mathrm{C}$ is mostly attributed to the loss of sodium which occurs at temperatures higher than $400{ }^{\circ} \mathrm{C}$. Small amounts of unreacted $\mathrm{Na}_{2} \mathrm{CO}_{3}$, which has been used as a starting material, may also contribute to the weight loss in this temperature region. 
The real and imaginary part of the dielectric permittivity $\beta-\mathrm{NaMnO}_{2}$ reveals two dielectric anomalies, at $90 \mathrm{~K}$ and 200 $\mathrm{K}$, as shown in figure 4. Two $\beta-\mathrm{NaMnO}_{2}$ pellets both synthesized with the same synthetic protocol have been measured upon heating with $500 \mathrm{kHz}$ and $100 \mathrm{kHz}$ applied in the measurement shown in figure $4 \mathrm{a}$ and $4 \mathrm{~b}$, respectively. The dielectric permittivity measurement shown in figure $4 \mathrm{a}$ was carried out using an electric field of $700 \mathrm{~V} / \mathrm{m}$. This value of electric field is comparable with the $685 \mathrm{~V} / \mathrm{m}$ applied in the measurement shown with the red line in figure $4 \mathrm{~b}$. Dielectric anomalies are observed in both frequencies tested, namely in $100 \mathrm{kHz}$ and $500 \mathrm{kHz}$. Interestingly, the dielectric anomaly at $90 \mathrm{~K}$ is greatly enhanced when the applied electric field is doubled from $685 \mathrm{~V} / \mathrm{m}$ to $1370 \mathrm{~V} / \mathrm{m}$ as it is observed in the measurement represented with the blue line in figure $4 \mathrm{~b}$. On the contrary, no difference is observed in the hump of the $200 \mathrm{~K}$ when the electric field is increased.

A

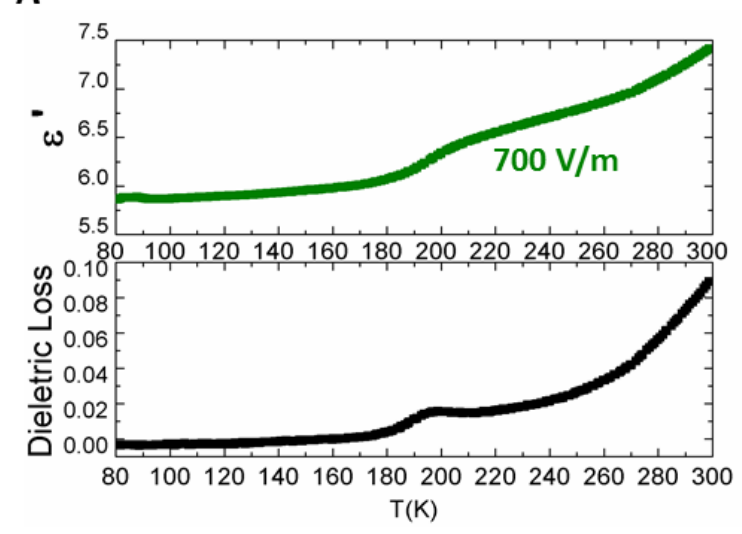

B

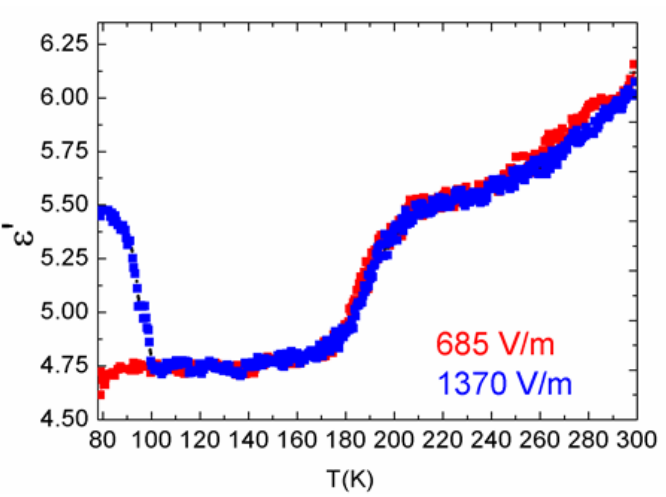

Figure 4. Real $\left(\varepsilon^{\prime}\right)$ and imaginary part $\left(\varepsilon^{\prime \prime}\right.$ or dielectric loss) under zero magnetic field on two pellets of $\beta-\mathrm{NaMnO}_{2}$ measured at the frequencies of $100 \mathrm{kHz}$ and $500 \mathrm{kHz}$ at $4 \mathrm{a}$ and $4 \mathrm{~b}$ figures, respectively. The dielectric anomaly at $90 \mathrm{~K}$ is majorly affected when the applied electric field is increased.

Neutron powder diffraction (NPD) data obtained at high resolution diffractometer BT1, shed light on the origin of the two dielectric anomalies that have been observed. Figure 5 shows temperature evolution of the square root (SQRT) of the normalized intensity versus d-spacing $(1.5 \AA-5.0 \AA)$ of the NPD patterns that have been obtained at the temperatures $300 \mathrm{~K}, 200 \mathrm{~K}, 150 \mathrm{~K}, 100 \mathrm{~K}$ and $50 \mathrm{~K}$. The blue and green ticks, shown in the NPD pattern of figure 5, correspond to the indexing of the crystal and magnetic structure, respectively. Anisotropic broadening, asymmetric and satellite peaks are the dominant characteristics of the NPD pattern indicating a rather complex crystal structure. Moreover, there are two striking characteristics in the NPD patterns at the magnetic Bragg peaks observed in the dspacing of $3.28 \AA$ and $4.6 \AA$ : a) The first one is the emergence of the peaks below $200 \mathrm{~K}$ which corresponds to a commensurate magnetic structure. At the same d-spacing, below $100 \mathrm{~K}$, at the pattern of $50 \mathrm{~K}$, satellite magnetic peaks appear, pointing towards the emergence of an incommensurate magnetic structure. The existence of dielectric anomalies at the same temperature regions where magnetic ordering appears is undoubtedly a signature of magnetodielectric coupling. The anomalies in the real part of the dielectric permittivity $\varepsilon^{\prime}$ correspond to the emergence of the polarization (since $\mathrm{P}=\varepsilon_{0}\left(\varepsilon^{\prime}-1\right) \mathrm{E}$, where $\mathrm{P}$ is the polarization, $\varepsilon_{0}$ is the dielectric permittivity of the vacuum, and $\mathrm{E}$ is the applied electric field). Previous studies in magnetoelectric compounds with the stoichiometry $\mathrm{ABO}_{2}$ such as $\mathrm{CuFeO}_{2}$ and $\mathrm{ACrO}_{2}{ }^{[11]-[12]}$ with triangular lattice topology of the magnetic cations have proved that the mechanism which generates the magnetodielectric coupling is connected with the magnetic frustration which leads to a complex spin configuration. 


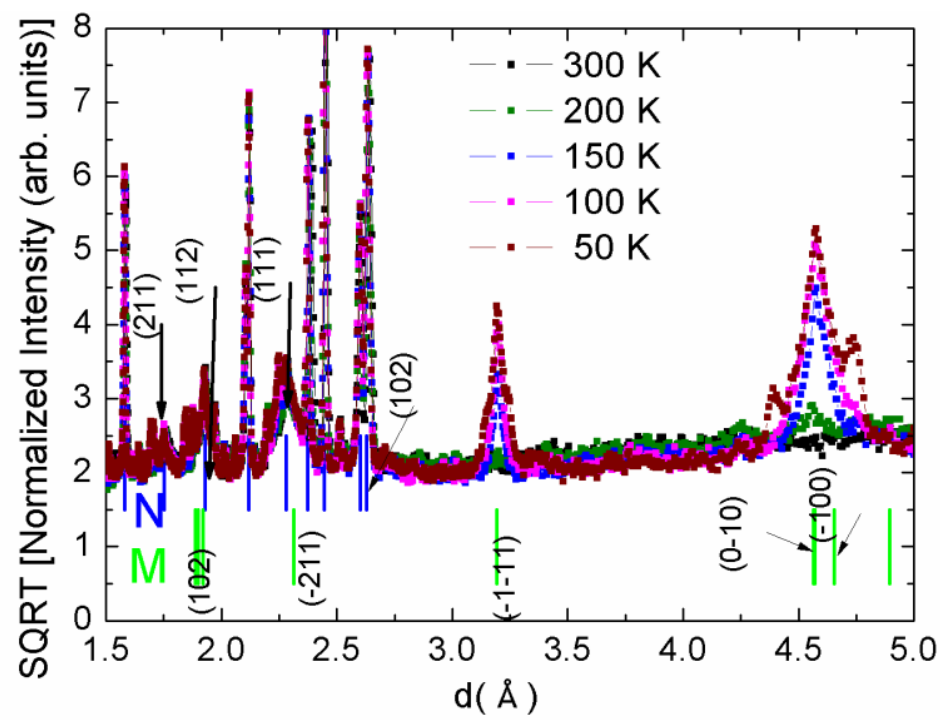

Figure 5. Neutron Powder Diffraction (NPD) patterns of the $\beta-\mathrm{NaMnO}_{2}$ obtained at different temperatures. Magnetic Bragg peaks emerge at $200 \mathrm{~K}$ and below $100 \mathrm{~K}$.
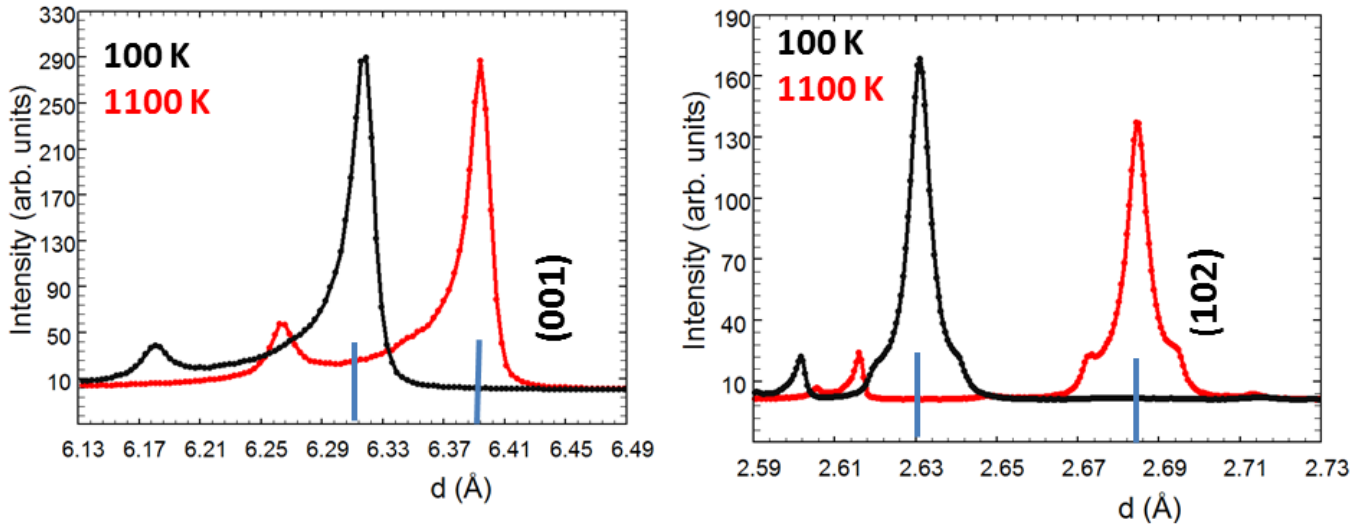

Figure 6. The 001 reflection on the left and 102 reflection on the right as shown on the patterns obtained at the 11BM syncthron of $\beta-\mathrm{NaMnO}_{2}$ reveal sattelite and asymetric peak at low (100 K- black line ) and high (1100 K-red line) temperatures.

To further explore the crystal structure, high resolution diffraction experiments were carried out in the11BM synchrotron on the temperature range between $100 \mathrm{~K}-1100 \mathrm{~K}$ (figure 6). Surprisingly, the broadening of the peaks, as well as the satellites and asymmetric peaks remain at temperatures even as high as $1100 \mathrm{~K}$. The analysis of the $11 \mathrm{BM}$ data, on the basis of a modulated structure (superstructure) gave no acceptable results, since the satellites of the (102) peak could not be indexed. These two unexplained peaks are so close to the main reflection that a corresponding vector would be too short in the reciprocal space to account for some structural modulation in the crystal. The synchrotron results and the relevant analysis point towards the existence of irregularities in the building diffraction domains which should be checked with electron diffraction. 


\section{CONCLUSIONS}

Magnetodielectric coupling of polycrystalline $\beta-\mathrm{NaMnO}_{2}$ is reported. The coupling is evident by the appearance of two anomalies in the dielectric permittivity around $90 \mathrm{~K}$ and at $200 \mathrm{~K}$, temperature regions in which according to the neutron diffraction patterns incommensurate and commensurate magnetic ordering develops. Increasing the applied electric field greatly affects the low temperature dielectric anomaly. A complete understanding of the mechanis $m$ that generates the magnetoelectric coupling requires the knowledge of the crystal and magnetic structure. The crystal structure shows irregularities which are observed in the neutron and synchrotron diffraction patterns. A complete explanation for the observed crystal structure complexity requires the observation of the $\beta-\mathrm{NaMnO}_{2}$ by electron diffraction and high resolution transmission electron microscopy, which are ongoing experiments.

\section{ACKNOWLEDGEMENTS}

This research has been co-financed by the European Union and Greek national funds through the Research Funding Program Heracleitus II (grand number 349309 WP1.56). We would also like to thank NCNR-NIST for providing beam time at the BT1 diffractometer. We are grateful to Prof. Vlacav Petricek for helpful discussions regarding the analysis of the $11 \mathrm{BM}$ synchrotron data.

\section{REFERENCES}

[1] Dzyaloshinskii, I., E., "On the magneto-electrical effect in antiferromagnetics" Sov. Phys. JETP 10, 628 (1959).

[2] Astrov, D., N., "The magnetoelectric effect in antiferromagnetics" Sov. Phys.JETP 11, 708 (1960).

[3] Spaldin, N., Fiebig, M., "The Renaisanse of the Magnetoelectric Effect" Science 309, 391-392 (2005).

[4] Eerenstein, W., Murther, N., D., Scott, J., F., "Multiferroic and magnetoelectric materials" Nature 442, 759-765 (2006).

[5] Sergienko, I., A., Dagotto, E., "Role of the Dzyaloshinskii-Moriya interaction in multiferroic perovskites" Phys. Rev. B 73, 094434 (2006).

[6] Van Aken, B., B., Palstra, T., M., Filippetti, A., Spaldin, N., A., "The origin of ferroelectricity in magnetoelectric $\mathrm{YMnO}_{3}$ " Nature Materials 3, 164-170 (2004).

[7] Khomskii, D., "Classifying multiferroics: Mechanisms and effects" Physics 2, 20 (2009).

[8] Wang, K., F., Liuab, J., M., Renc, Z., F., "Multiferroicity: the coupling between magnetic and polarization orders" Advances in Physics 58(4), 321-448 (2009).

[9] Arima, T., H., "Spin Driven Magneto-electric Effects in Frustrated Magnetic Systems" Journal of the Physical Society of Japan 80, 052001 (2011).

[10] Kimura T., J., C., Lashley, J., L., Ramirez, A., P., "Inversion-symmetry breaking in the noncollinear magnetic phase of the triangular-lattice antiferromagnet $\mathrm{CuFeO}_{2}$ " Phys.Rev.B 73, 220401R (2006).

[11] Seki, S., Onose, Y., Tokura, Y., "Spin-Driven Ferroelectricity in Triangular Lattice Antiferromagnets $\mathrm{ACrO}_{2}(\mathrm{~A}$ $\mathrm{Cu}, \mathrm{Ag}, \mathrm{Li}$, or Na)" Phys. Rev. Lett. 101, 067204 (2008).

[12] Terada, N., Khalyavin, D., D., Manuel, P., Tsujimoto, Y., Knight, L., Radaelli, P., G., Suzuki, H., S., Kitazawa, H., "Spiral-Spin-Driven Ferroelectricity in a Multiferroic Delafossite AgFeO," Phys. Rev. Lett. 109, 097203 (2012).

[13] Dzyaloshinsky, I. "A thermodynamic theory of weak ferromagnetism of antiferromagnetics" J. Phys. Chem. Solids, 4(4), 241-255 (1958).

[14] Moriya, T., “Anisotropic superexchange interaction and weak ferromagnetism” Phys. Rev. 120, 91 (1960).

[15] Katsura, H., Nagaosa, N., Balatsky A., B., "Spin Current and Magnetoelectric Effect in Noncollinear Magnets" Phys. Rev. Lett. 95, 057205 (2005).

[16] Arima, T., H.,“ Ferroelectricity Induced by Proper-Screw Type Magnetic Order” J. Phys. Soc. Jpn. 76 (7), 073702 (2007).

[17] Jia, C., Onoda, S., Nagaosa, N., Han, J., H., "Bond electronic polarization induced by spin" Phys. Rev. B 74, 224444 (2006).

[18] Parant, J., P., Olazcuaga, R., Devallete, M., Fouassier, C., Hagenmuller, P., "Sur Quelques Nouvelles Phases de Formule $\mathrm{Na}_{\mathrm{x}} \mathrm{MnO},(\mathrm{x} \leq 1)$ " J. Solid State Chem. 3, 1-11 (1971).

[19] Abou El Sherbini, Kh., S., Askara, M., H., Schollhorn, R.., "Hydrated layered manganese dioxide Part I. Synthesis and characterization of some hydrated layered manganese dioxides from $\alpha-\mathrm{NaMnO}_{2}$ " Solid State Ionics $150,407-415$ (2002). 
[20]Abou-El-Sherbini, Kh., S., Askara, M., H., Schollhorn, R.., "Hydrated layered manganese dioxide Part II. Electrochemical behaviour of some hydrated layered manganese dioxides in alkaline electrolytes" Solid State Ionics 150, 417- 430 (2002).

[21] Abou-El-Sherbini, Kh., S., Askara, M., H., Schollhorn, R.., "Hydrated layered manganese dioxide: III. Role of bismuth oxide on the redox behavior of hydrated layered manganese dioxides" Solid State Ionics 139, 121-133 (2001).

[22] Kruk, I., Zajdel, P., Beek, W., Bakaimi, I., Lappas, A., Stock, C., Green, M., A., "Coupled Commensurate Cation and Charge Modulation in the Tunneled Structure Na 0.40(2) $\mathrm{MnO}_{2}$ " J. Am. Chem. Soc. 133, 13950-13956 (2011).

[23] Doeff, M., M., Richardson, T., J., Hwang K., T., "Electrochemical and structural characterization of titaniumsubstituted manganese oxides based on $\mathrm{Na}_{0.44} \mathrm{MnO}_{2}$ " Journal of Power Sources 135, 240-248 (2004).

[24]. Doeff, M., M., Richardson, T., J., Hwang K., T., "Orthorhombic Na x MnO2 as a Cathode Material for Secondary Sodium and Lithium Polymer Batteries" J. Electrochem.Soc. 141 (11), L145-L147 (1994).

[25] Doeff, M., M.; Richardson, T. J.; Kepley, L., "Lithium Insertion Processes of Orthorhombic $\mathrm{Na}_{x} \mathrm{MnO}_{2}-\mathrm{Based}$ Electrode Materials” J. Electrochem. Soc. 143 (8), 2507-2516 (1996).

[26] Giot, M., Chapon, L., C., Androulakis, J., Green, M., A., Radaelli, P., G., Lappas, A., "Magnetoelastic Coupling and Symmetry Breaking in the Frustrated Antiferromagnet $\alpha-\mathrm{NaMnO}_{2}$ " Phys. Rev. Lett. 99, 247211 (2007).

[27] Zorko, A., El Shawish, S., Arčon, D., "Magnetic interactions in $\alpha-\mathrm{NaMnO}_{2}$ : Quantum spin-2 system on a spatially anisotropic two-dimensional triangular lattice" Phys. Rev. B 77, 024412 (2008).

[28] Stock, C., Chapon L., C., Adamopoulos, O., Lappas, A., Giot, M., Taylor, J., W., Green, M., A., Brown, C., M., Radaelli, P., G., "One-Dimensional Magnetic Fluctuations in the Spin-2 Triangular Lattice $\alpha-\mathrm{NaMnO}_{2}$ " Phys. Rev. Lett. 103, 077202 (2009). 